\title{
Esforço percebido durante o treinamento intervalado na natação em intensidades abaixo e acima da velocidade crítica
}

\author{
Flavio G. Suzuki ${ }^{1}$ \\ Nilo M. Okuno ${ }^{1}$ \\ Adriano E. Lima-Silva 3 \\ Luiz A. B. Perandini 1 \\ Eduardo Kokubun 4 \\ Fábio Y. Nakamura 1,2
}

https://doi.org/10.5628/rpcd.07.03.299

\author{
${ }^{1} \mathrm{G}$ rupo de Estudo das A daptações Fisiológicas ao \\ Treinamento (GEAFIT). Centro de Educação Física e \\ Desportos, Universidade Estadual de Londrina, Brasil \\ 2 Grupo de Estudo e Pesquisa em M etabolismo, Nutrição \\ e Exercício. Centro de Educação Física e D esportos. \\ Universidade Estadual de Londrina, Brasil \\ ${ }_{3}$ Laboratório de Pesquisa M orfo-Funcional, U niversidade \\ do Estado de Santa Catarina, Brasil \\ 4 Instituto de Biociências, Departamento de Educação \\ Física, Universidade Estadual Paulista, Brasil
}

\section{ABSTRACT \\ Perceived exertion during swimming interval training at intensi- ties below and above critical velocity}

intensidades na resposta de esforco os efeitos das pausas edas mento intervalado na natação. Oito indivíduos realizaram inicialmente repetições de 100, 200 e 400 m para a determinação da velocidade crítica (VC) e capacidade de trabal ho anaeróbio (CTA). Em outras ocasiões, os sujeitos foram submetidos a quatro sessões de treinamento intervalado realizando repetições de $200 \mathrm{~m}$. Foram realizadas duas sessões a 95\% e outras duas a $110 \%$ da VC. A única diferença entre as sessões na mesma intensidade de exercício foram os intervalos das pausas, de 20 ou 40 s. Ao final de cada repetição de 200 m, os participantes reportavam o esforço percebido por meio da escala de Borg. A $95 \%$ da VC não foi verificada diferença significativa no comportamento do esforço percebido entre os regimes de pausas de 20 e 40 s. Em contraste, a $110 \%$ da VC houve diferenças significativas no esforço percebido reportado e no número de repetições realizadas ( $40 \mathrm{~s}=5,7 \pm 2,1$ repetições; $20 \mathrm{~s}=4,0$ $\pm 1,0$ repetições) entre os regimes de pausas. Dessa forma, 0 possível mecanismo explicativo para as respostas do esforço percebido abaixo e acima da VC em diferentes regimes de pausas parece ser a utilização ou não da CTA.

Palavras-chave: natação, velocidade crítica, esforço percebido.
The aim of the present study was to verify the effects of rest intervals and intensities in perceived exertion responses during swimming interval training. Eight individuals performed initially 100, 200 and 400 $\mathrm{m}$ bouts for the critical velocity (VC) and anaerobic work capacity (CTA) estimation. A dditionally the subjects were submitted to four sessions of interval training comprised by $200 \mathrm{~m}$ bouts. It was conducted in two sessions at $95 \%$ and $110 \%$ of VC. The only difference between the sessions at the same intensity of exercise was the rest intervals of 20 or $40 \mathrm{~s}$. At the final of each $200 \mathrm{~m}$ bout, the participants reported significant difference in perceived exertion responses between the 20 and $40 \mathrm{~s}$ rest intervals regimes. Nonetheless, at $110 \%$ of VC there were significant differences in the perceived exertion reported and in number of performed bouts $(40 \mathrm{~s}=5.7 \pm 2.1$ bouts; $20 \mathrm{~s}=4.0 \pm 1.0$ bouts) between the rest intervals regimens. Therefore, the possible mechanism for the perceived exertion responses below and above the VC under different rest intervals regimens seems to be the utilization or not of CTA. the perceived exertion through Borg scale. At $95 \%$ of VC there was not

Key-words: swimming, critical velocity, perceived exertion. 


\section{INTRODUÇÃO}

Trabalhos de Wakayoshi et al. $(32,33,34)$ foram os primeiros a aplicar o modelo de velocidade crítica $(17,18)$ à natação e a investigar seu significado fisiológico. Nesse contexto, a determinação do parâmetro foi realizada em swimming flume(32). Wakayoshi et al. $(33,34)$ e outros $(8,10,15)$ também testaram protocolos em piscinas. Não foram encontradas diferenças entre os valores de velocidade crítica (VC) determinados nas duas formas de nado(33). Em adição, a VC estava altamente correlacionada com o limiar anaeróbio obtido por concentração fixa de 4 mM de lactato sangüíneo(15,32,33,34), além de se aproximar da velocidade de nado com máximo estado estável de lactato(15,34).

Do ponto de vista prático, técnicos e atletas freqüentemente não têm acesso a medidas de VC e respostas de lactato ao exercício. Dessa forma, prescrevem os treinamentos em função de percentuais de desempenhos máximos nas distâncias, e/ ou com base na percepção subjetiva do atleta.

Tradicionalmente, o esforço percebido apresenta relação direta simples com as intensidades de exercício(5). Recentemente, atribuiu-se ao esforço percebido um papel relevante na modulação da tolerância ao exercício. Essa variável é entendida como uma representação central, complexa e integrada(6), das diversas funções corporais modificadas agudamente pela atividade muscular(30), sendo também influenciada por experiências de exercício acumuladas no passado(1). Assim, o esforço percebido pode ser fonte de informação importante para apontar os limites de tolerância ao esforço, protegendo de danos sistemas fisiológicos importantes, como o cardiovascular(22). Caso essa resposta possa ser relacionada a parâmetros de sobrecarga, as escalas de percepção de esforço poderiam ser utilizadas com mais freqüência no contexto do planejamento e prescrição do treinamento de nadadores.

Combinando os pressupostos do modelo de velocidade crítica com as noções mais atuais de esforço percebido, foi proposto, recentemente, o limiar de esforço percebido (LEP)(19,20). Em tese, o LEP corresponde ao máximo estado estável de percepção de esforço, além de apresentar alta correlação com a potência crítica, VC e com um indicador do máximo estado estável do consumo de oxigênio(19,20), sendo desta forma um demarcador de transição metabólica entre o domínio intenso e severo de exercício(9). Adicionalmente, foi observado nesses dois estudos que em intensidades superiores a VC, o esforço percebido aumentava em função do tempo a uma taxa proporcional à intensidade. Uma possível explicação para o fenômeno seria que o esforço percebido nas intensidades severas( ${ }^{(9)}$ seria regulado pela velocidade de utilização da capacidade de trabal ho anaeróbio (CTA). Esse segundo parâmetro do modelo é representativo da capaci dade de transferência energética a partir dos estoques de fosfagênios e da glicólise anaeróbia(7,17,18). Assim, o esforço percebido em intensidades acima da VC seria uma conseqüência dos distúrbios periféricos associados à depleção de fosfagênios e acidose metabólica, concomitante à mobilização crescente da atividade neuromotora eferente(6). Portanto, uma previsão lógica dessas interpretações recentes do modelo, é a ausência de aumento do esforço percebido em cargas retangulares ou intervaladas abaixo da VC, porque nessas condições a CTA não seria modificada. Cabe ressaltar que outros fatores não fisiológicos, relacionados a fatores psicológicos e situacionais, bem como expectativas com relação à duração e/ ou distância a serem percorridas, podem afetar a percepção subjetiva de esforço(1,2,27). Isso motivou alguns autores a proporem escal as subjetivas de estimativas de tempo remanescente de esforço.

Foi elaborada a hipótese de que treinamentos intervalados realizados em intensi dades inferiores à VC seriam completadas sem alterações significativas no esforço percebido ao longo do tempo, uma vez que elas estariam associadas a intensidades inferiores ao LEP, e que a duração das pausas entre as repetições não influenciaria nessa resposta psicofísica, já que a CTA não seria utilizada. Já em intensidades maiores que a VC, a mobilização da CTA levaria à ausência de estado estável nas respostas de esforço percebido, já que estariam associadas a intensidades superiores ao LEP. Além disso, pausas mais curtas entre os esforços ocasionariam mai ores respostas perceptivas do que pausas mais longas. Isso porque, nessas condições mais severas de intensidade, o grau de utilização acumulado de CTA, sem a devida recuperação, deve interferir na modulação da tolerância ao esforço na natação. 
Dessa forma, foi objetivo deste trabal ho verificar os efeitos das pausas e das intensidades na resposta de esforço percebido durante treinamento intervalado na natação. A confirmação das hipóteses mencionadas acima permitiria a proposição de novas interpretações acerca do significado fisiológico e das inter-relações teóricas entre os parâmetros do modelo de velocidade crítica e respostas de esforço percebido no exercício.

\section{MÉTODOS}

\section{Sujeitos}

Fizeram parte deste estudo oito adolescentes do sexo masculino $(n=6)$ e feminino $(n=2)$, com 15,6 $\pm 0,5$ anos de idade, que participavam de competições de nível estadual e treinavam cinco vezes por semana. A experiência dos atletas em competições variava entre dois e cinco anos. Todos os participantes e seus responsáveis foram informados sobre os procedimentos do estudo e assinaram termo de consentimento livre e esclarecido aprovado pelo Comitê de Ética em Pesquisa da Universidade Estadual de Londrina.

\section{Determinação dos parâmetros do} modelo de velocidade crítica

Previamente aos testes para determi nação dos parâmetros do modelo de velocidade crítica, todos os sujeitos realizaram aquecimento habitual sem controle direto dos investigadores. $\mathrm{O}$ aquecimento não foi monitorado pelos avaliadores, pois foi o técnico quem aplicou o aquecimento diário para os treinamentos. Após o aquecimento, os sujeitos foram instruídos a reportar o momento em que se sentiam preparados para iniciar cada teste. A piscina em que o estudo foi conduzido tinha $25 \mathrm{~m}$ de comprimento. As distâncias utilizadas para os testes foram de 100 , 200 e $400 \mathrm{~m}^{(11,16)}$. Houve al eatoriedade na ordem de imposi ção dos testes, os quais foram aplicados em dias diferentes, e individual mente. A orientação era para que os nadadores percorressem as distâncias no menor tempo possível. O tempo do desempenho foi registrado por meio de um cronômetro manual, sendo realizada a aproximação em décimos de segundo.

Para estimativa da VC e CTA foi utilizada a equação: [Tempo = (Distância - CTA $) /$ VC]. A equação $($ Distância $=$ VC $x$ tempo + CTA $)$ não foi utilizada pois segundo pressupostos matemáticos, a variável independente deve ser colocada no eixo $x$. Dessa forma, as variáveis foram realocadas, de modo a distância ficar no eixo x. Os parâmetros foram calculados por meio de regressão linear.

Treinamentos intervalados abaixo e acima da velocidade crítica Após terem a VC e CTA estimadas, os nadadores realizaram mais quatro sessões de testes. Essas quatro sessões foram treinamentos intervalados, da seguinte forma: (A) duas sessões com intensidade equival ente a $95 \%$ da VC (abaixo da VC) e (B) duas sessões com intensidade equivalente a $110 \%$ da VC (acima da VC). Tanto em (A) quanto em (B), solicitou-se que os participantes realizassem o mai or número possível de repetições de $200 \mathrm{~m}$ na velocidade pré-estabelecida, com 20 ou 40 s de pausas fixas entre os esforços. A seqüência dos treinamentos foi aleatória, por meio de sorteio a priori da ordem a ser seguida. Tanto em (A) como em (B) os participantes realizaram até dez repetições.

O controle da velocidade se deu por meio de sinais sonoros emitidos pelo avaliador durante todo o percurso, mas, sobretudo nas viradas, com informações adicionais real izadas com gestos manuais referentes às diferenças entre o tempo real e o previsto, para que a duração final das repetições se aproximasse dos tempos a serem atingidos. As informações realizadas por gestos manuais eram obtidas pelo nadador na primei ra respiração realizada após o impulso da virada. A faixa de tolerância para erros no ritmo era de até $92,5 \%$ da VC em (A), e de $105 \%$ da VC em (B). Essas faixas de tolerância foram adotadas para que não houvesse uma grande variação na intensidade dos testes, na qual poderia comprometer a análise dos dados quando comparado os regimes de 20 e 40 s. Não houve necessidade de controle da faixa superior de erro porque raramente os nadadores realizavam as repetições em intensidades mais altas que as pré-definidas, com exceção da primeira, em todos os treinamentos. Em (B), o limite inferior foi menos rigoroso porque a manutenção da velocidade prédeterminada era mais difícil, em função da alta intensidade. Depois de cada repetição, era dada a informação do tempo total despendido na distância, para que o nadador pudesse real izar ajustes na 
intensidade nas repetições subseqüentes em caso de erro. Caso repetisse performances aquém da faixa de tolerância por duas vezes consecutivas, o treinamento era interrompido, e a última repetição era excluída da análise posterior.

Ao final de cada repetição de $200 \mathrm{~m}$, os participantes reportavam o esforço percebi do por meio da escala de Borg de 15 pontos(5). Cabe ressaltar que os participantes não conheciam as hipóteses que estavam sendo testadas, mas tinham conhecimento de qual era o regime de treinamento intervalado a ser realizado no dia.

Tratamento estatístico

Os resultados de performance nos esforços máximos de 100 m, 200 m e 400 m, assim como a VC e a CTA, foram reportados mediante estatística descritiva. Anteriormente a análise dos dados, foi realizado o teste Kolmogorov-Smirnov (com correção de Lilliefor's) a fim de verificar a normalidade dos dados. A comparação entre as respostas de esforço percebido durante os treinamentos intervalados, tanto a $95 \%$ quanto a $110 \%$ da VC, foi realizada a partir de ANOVA two-way para medidas repetidas, tendo como fatores regime de pausas ( 20 ou $40 \mathrm{~s}$ ) e seqüência de repetições, e como variáveis dependentes tempos ou esforço percebido. O teste post hoc de Newman-Keuls foi utilizado para a identificação das diferenças pontuais entre as médias. Foi adotado teste t para amostras pareadas na comparação do número de repetições real izadas em cada regime de pausas, separadamente nas duas intensidades a serem atingidas. $O$ nível de significância préestabelecido em todas as análises foi de $p<0,05$.

\section{RESULTADOS}

As médias dos tempos da amostra estudada para as distâncias de 100 m, 200 m e 400 m foram respectivamente de $76,8 \pm 12,1 ; 174,7 \pm 31,3$ e $379,1 \pm 70,0$ s. Os valores médios dos parâmetros VC e CTA dos nadadores foram iguais a $1,00 \pm 0,17 \mathrm{~m} \cdot \mathrm{s}^{-1}$ e $24,6 \pm$ $4,5 \mathrm{~m}$, respectivamente. $O$ valor médio de $R^{2}$ foi de $1,0 \pm 0,0$, mostrando bom ajuste dos dados de performance à equação prevista pelo modelo. A Figura 1 mostra o ajuste da equação prevista pelo modelo de velocidade crítica aos resultados de um nadador da amostra.

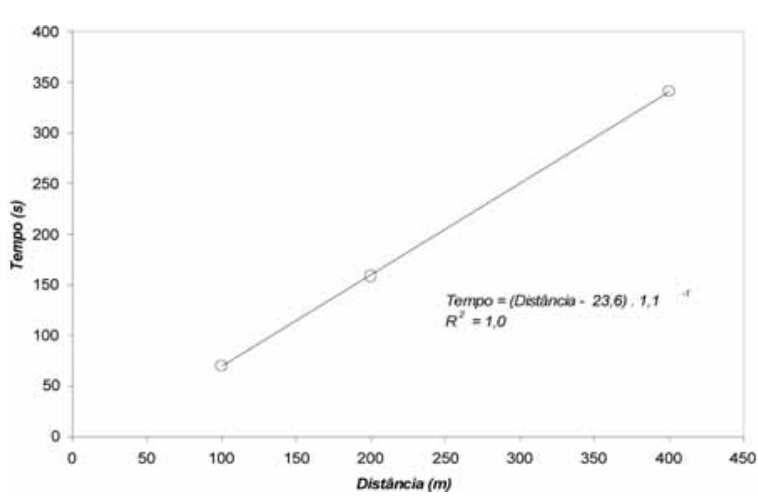

Figura 1. Relação distância-tempo de um nadador pertencente à amostra.

Conforme mostrado na Figura 2, houve estabilidade do tempo necessário para realização das repetições de $200 \mathrm{~m}$ em intensidade equivalente a $95 \%$ da VC. Apenas um indivíduo não foi capaz de cumprir a meta de dez repetições, interrompendo, tanto com pausas de $20 \mathrm{~s}$ quanto com pausas de $40 \mathrm{~s}$, o treinamento intervalado na 5 a repetição. Esse sujeito foi eliminado da análise nessa etapa do estudo, por não acompanhar o comportamento do restante do grupo. Em ambas as situações, a 1a repetição foi realizada abaixo do tempo previsto. Todavia, nas repetições subseqüentes os atletas conseguiram desempenhar bastante próximos da intensidade a ser atingida.

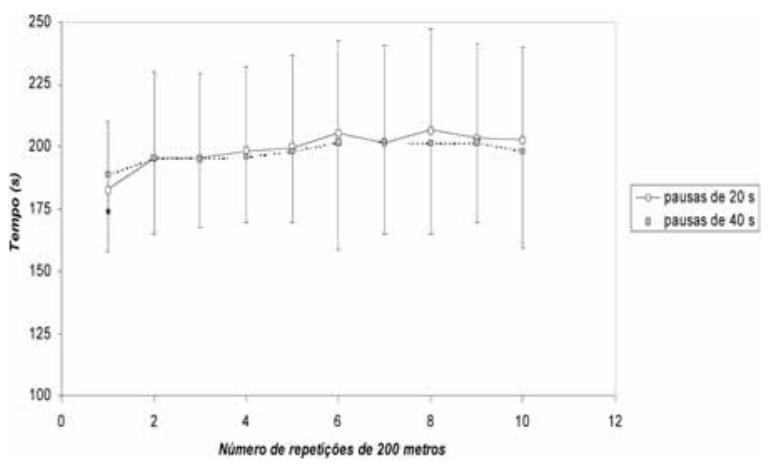

Figura 2 - Tempos nas repetições de $200 \mathrm{~m}$ realizados a $95 \%$ da VC, com 20 e $40 \mathrm{~s}$ de pausas.

* diferença significante $(p<0,05)$ comparadas as repetições seguintes.

$\mathrm{Na}$ Figura 3, observa-se que não houve estabilidade do desempenho nas repetições acima da VC. No entanto, uma diferença significante entre os regimes de pausas só ocorreu na 3 a repetição. A análise foi 
limitada estatisticamente às três primeiras repetições porque parte dos nadadores não conseguiu realizar os esforços seguintes. Os tempos nas últimas repetições, associados à exaustão dentro de cada protocolo, não foram diferentes ( $p>0,05)$ quando comparados os treinamentos com 20 s $(202,4 \pm 38,1$ s) e $40 \mathrm{~s}$

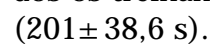

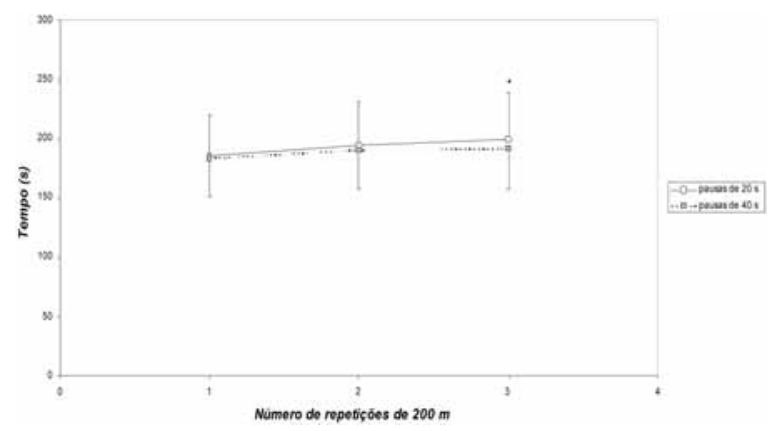

Figura 3. Tempos nas repetições de $200 \mathrm{~m}$ realizadas a $110 \%$ da VC, com 20 e $40 \mathrm{~s}$ de pausas.

* diferença significante $(p<0,05)$ na repetição entre os regimes de pausas. Obs.: Houve diferença significante entre todos as repetições realizadas dentro do mesmo regime de pausas, com exceção da $2^{a}$ para a $3^{a}$ repetição do regime com pausas de $40 \mathrm{~s}$.

Com relação ao esforço percebido, não houve diferenças $(P>0,05)$ entre os regimes de pausas de $20 \mathrm{~s}$ e de 40 s entre as repetições conduzidas a $95 \%$ da VC, apesar de tendência visual para que com pausas mais longas o esforço percebido fosse menor (figura 4). Não houve estado estável de esforço percebido ao longo das repetições abaixo da VC. A tendência era que os valores da segunda metade do treinamento fossem maiores que os valores da primeira metade.

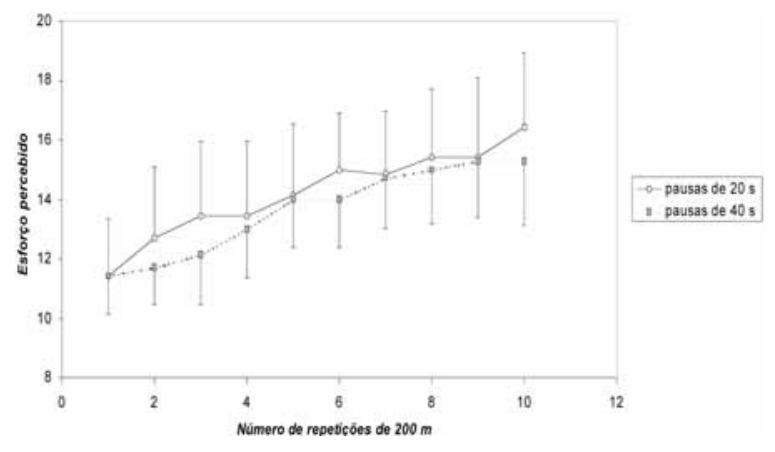

Figura 4. Comportamento do esforço percebido em repetições de $200 \mathrm{~m}$ realizadas a 95\%da VC, com 20 e $40 \mathrm{~s}$ de pausas.

Obs.: Não houve diferença significante entre os regimes de pausas em nenhuma repetição.
A $110 \%$ da VC, em contraste com a intensidade abaixo da VC, houve diferenças $(p<0,05)$ no esforço percebido reportado entre os regimes de pausas em todos as repetições (Figura 5). Além disso, novamente, não houve estado estável do esforço percebido em quaisquer das condições.

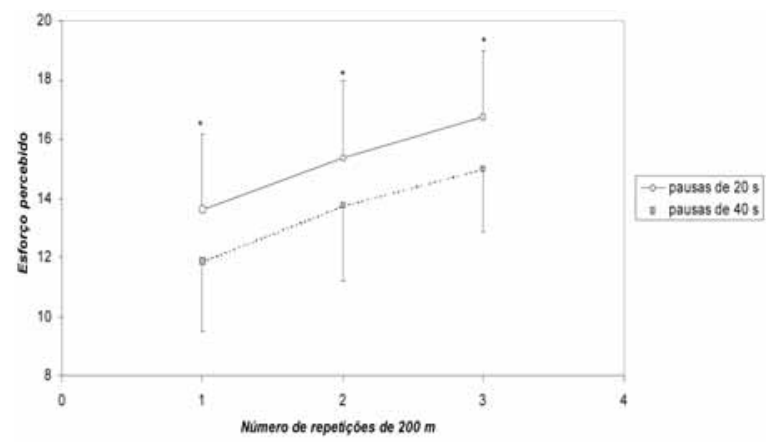

Figura 5- Comportamento do esforço percebido em repetições de $200 \mathrm{~m}$ realizadas a $110 \%$ da VC, com 20 e 40 s de pausas. * diferença significante $(p<0,05)$ em as repetições equivalentes, com $40 \mathrm{~s}$ de pausa.

Obs.: Houve diferença significante $(P<0,05)$ entre todos as repetições dentro do mesmo regime de pausas.

Na comparação do número de repetições realizadas em intensidade acima da VC em cada regime de pausa (Tabela 1), houve diferença significante $(p<0,05)$, sendo maior com 40 s $(5,7 \pm 2,1$ repetições) do que com 20 s ( $4,0 \pm 1,0$ repetições).

Tabela 1. Número de repetições de $200 \mathrm{~m}$ em intensidades acima da VC com pausas de 20 e 40 s, para todos os sujeitos.

\begin{tabular}{|c|c|c|c|c|c|c|c|}
\hline \multirow[t]{3}{*}{ Pausas } & \multicolumn{7}{|c|}{ Número de repetições de $200 \mathrm{~m}$} \\
\hline & Sujei & ujeit & Sujeit & Sujei & Sujei & Sujei & ujeito \\
\hline & 1 & 2 & 3 & 4 & 5 & 6 & 7 \\
\hline $20 \mathrm{~s}$ & 3 & 5 & 3 & 5 & 3 & 4 & 5 \\
\hline $40 \mathrm{~s}$ & 3 & 7 & 3 & 7 & 8 & 7 & 5 \\
\hline
\end{tabular}

\section{DISCUSSÃO}

Nossos resultados apontaram que dos oito componentes da amostra estudada, sete conseguiram percorrer um total de $2000 \mathrm{~m}$ ( $10 \times 200 \mathrm{~m}$ ) sem exaustão em intensidade abaixo da VC (95\%), e sem diferenças nas respostas de esforço percebido entre as repetições correspondentes, com pausas de 20 e $40 \mathrm{~s}$. A explicação desses resultados se deve à VC ser considerada a maior intensidade que pode ser mantida 
por um longo período de tempo sem a ocorrência da fadiga(17). Além disso, essa variável está relacionada ao máximo estado estável de lactato e consumo de oxigênio(15,18,29,34). Quando o indivíduo realiza exercícios acima da VC, há a utilização da CTA para complementar a demanda necessária do exercício que não consegue ser suprida pelo sistema aeróbio, e quando ocorre o esgotamento dessa reserva, o indivíduo entra em exaustão. Alguns estudos têm demonstrado que CTA está relacionada ao máximo déficit acumulado de oxigênio(7,14). Esses pressupostos confirmam parte das previsões inicialmente delineadas para este estudo baseadas em nossos estudos recentes $(19,20)$, em que observamos que a taxa de aumento do esforço percebido, em cargas severas de trabaIho(9), era proporcional à intensidade do exercício. A extrapolação da relação linear entre a taxa de aumento do esforço percebido (ordenada) com a intensidade do exercício (abscissa) forneceu um intercepto no eixo $\mathrm{x}$ em que, teoricamente, o esforço percebido teria seu máximo estado estável. Essa intensidade, correspondente ao LEP, o qual não foi diferente da VC e potência crítica(19,20). Dessa forma, a nossa hipótese de explicação era de que a CTA seria um modulador sensorial e perceptivo dos limites de tole rância do exercício. Em situações de utilização da CTA, o esforço percebi do aumentaria até o indivíduo entrar em exaustão. Como em intensidade abaixo da VC (95\%) não há utilização da CTA, diferentes tempos de pausas entre as repetições teoricamente não influenciariam na resposta perceptiva dos indivíduos. Já em relação à intensidade de $110 \%$ da VC, os resultados mostraram que com $20 \mathrm{~s}$ de pausa entre as repetições o esforço percebido era significantemente mai or nas três primeiras repetições, quando comparado com os valores obtidos no treinamento intervalado com $40 \mathrm{~s}$ de pausa.

O mecanismo responsável pelo comportamento do esforço percebido na condição supracitada seria a relação diferenciada entre utilização e reposição da CTA. De acordo com o modelo de LEP, a taxa de aumento do esforço percebido seria proporcional à mobilização do metabolismo anaeróbio (acúmulo de $\mathrm{H}^{+}$, depleção de fosfagênios), e em pausas menores de treinamento interval ado acima da VC, a recuperação da CTA seria menor. É provável que esse fato tenha determinado o comportamento do esforço percebido nas situações de $20 \mathrm{~s}$ e $40 \mathrm{~s}$ de pausas, e também na tolerância ao esforço em ambas as condições ( $20 \mathrm{~s}=4,0 \pm 1,0$ repetições; 40 $s=5,7 \pm 2,1$ repetições).

Trabal ho recente de Seiler e Hetlelid(27) demonstra ram que corredores bem treinados submetidos a treinamento intervalado em esteira, objetivando a manutenção da maior velocidade média possível durante $6 \times 4$ min, não apresentaram diferenças de esforço percebido em cada uma das repetições quando eram permitidas pausas de um, dois, ou quatro minutos entre os esforços. Eles sustentaram velocidades médias entre $83-85 \%$ da velocidade associada ao $\mathrm{VO}_{2}$ max. É provável que essa intensidade tenha sido escolhida em todos os regimes de pausa por se aproximar da intensidade de limiar anaeróbio(29), ou VC.

A questão principal a ser investigada seria a origem dos estímulos aferentes de aumento do esforço percebido nessa condição de ausência de mobilização da CTA. Estudo de Baldwin et al.(4) apresenta evidências de que o construto governador central poderia atuar nessa condição, sendo a principal fonte de retroalimentação e integração central um mecanismo de glicostato(26). Ele teria propriedade de monitorar os estoques musculares de glicogênio e modular o ritmo de exercício em atividades com metas de distâncias fixas. Assumiu-se que as reservas de glicogênio não seriam repostas nos interval os de pausas de 20 ou 40 s. A reposição parece demandar minutos (até uma hora) para ocorrer de forma significativa(14).

Dessa forma, a utilização (acima da VC) ou não (abaixo da VC) da CTA parece modular as respostas perceptivas aos exercícios intervalados.

Particularmente na $1^{1}$ a repetição do treinamento acima da VC, não se esperava diferença no esforço percebido (figura 5), já que os tempos não diferiram (figura 3). Uma explicação provável para o fenômeno seria que os nadadores sabiam a priori a intensidade e o regime de pausas a que seriam submetidos. Assim, por experiência prévia(1), teriam condições de elaborar resposta antecipatória (feed forward), "forçando" a maiores níveis de percepção de esforço no regime mais pesado em função das menores pausas (20 s). Neste estudo, tanto na intensidade abaixo da VC quanto acima da VC, estabeleceu-se um limite superior de dez repetições de $\mathbf{2 0 0}$ m, que perfaziam um 
total de $2000 \mathrm{~m}$. Esse limite foi estabelecido para não sobrecarregar excessivamente os atletas avaliados. Contudo, abaixo da VC, praticamente todos os componentes da amostra conseguiram completar as dez repetições. Já acima da VC, nenhum deles conseguiu completar mais de oito repetições, sendo a média de repetições menor com $20 \mathrm{~s}$ de pausa entre os esforços do que com $40 \mathrm{~s}$ ( $20 \mathrm{~s}$ : 4,0 $0 \pm 1,0$ repeti-

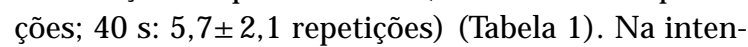
sidade acima da VC, a exaustão ocorreu por falta de condições para que o ritmo pré-estabelecido pudesse ser mantido pelo nadador. Dessa forma, o critério para finalização das séries nas diferentes intensidades foi diferente.

Segundo trabalho recente de Baden et al.(3), as respostas perceptivas ao esforço são dependentes da forma como se dá o estabelecimento de meta para sua final ização. Nesse estudo, uma amostra foi levada a se exercitar sob três condições, todas a $75 \%$ da velocidade de pico obtida em protocolo incremental na esteira. Em uma das situações $(A)$, recebiam a instrução de que correriam por 20 min nessa intensidade. Em outra (B), não havia informação sobre a duração, mas ela se mantinha em 20 min. Na terceira situação $(C)$, os sujeitos eram enganados, porque recebiam a informação de que correriam por dez minutos, mas ao final desse período eram informados de que teriam que correr mais dez minutos. Entre o $10^{\circ} \mathrm{e} \mathrm{o} 11^{\circ}$ mi nutos, houve aumento abrupto do esforço percebido em (C), o qual persistiu até o $17^{\circ}$ minuto maior que em (A) e (B). Esse esforço percebido aumentado estava associado a experiências emocionais negativas como raiva, frustração e desconfiança. Isso mostra que a forma de estabelecimento de meta para o exercício pode interferir nas respostas perceptivas.

De acordo com UImer(31), sinais motores eferentes para os músculos em atividade não incluem apenas comandos de parâmetros espaço-temporais para o controle dos movimentos. Eles contêm também informações para controle extracelular da taxa metabólica sustentável no exercício. A realização freqüente de exercícios não propiciaria somente retenção de melhores padrões mecânicos de controle motor, mas também de padrões de regulação e controle dos ajustes agudos ao desgaste energético. Isso poderia evitar a fadiga precoce, ou perturbação danosa da homeostase.
Os mecanismos de controle central do ritmo de exercício foram coletivamente chamados de teleoantecipação(31). Os mecanismos de teleoantecipação, em tese, permitem a realização de projeções imediatas de performance tendo como base estimativas da reserva metabólica, taxa metabólica atual e a distância para a meta final. Atuam também as experiências prévias com exercício(1). Dessa forma, de acordo com o modelo de UImer(31), o esforço percebido pode ser visto como uma ferramenta útil para acessar as estratégias teleoanteci patórias empregadas pelo organismo. Além disso, pode permitir o estabel ecimento de critérios operacionais para definir al gumas variáveis do treinamento intervalado, sobretudo relacionadas à intensidade.

Em contraste com o modelo de teleoantecipação, que foi revisto e proposto pelo grupo de Noakes em torno do conceito de governador central, o de velocidade crítica se enquadra dentre os que Noakes e St Clair Gibson(25) consideram model os "catastróficos". Modelos "catastróficos" são aqueles que pressupõem que o limite de tolerância a esforços com diferentes características de intensidade/ duração é estabelecido por eventos bioquímicos de degradação de substratos (glicogênio, fosfagênios) e/ ou acúmulo de metabólitos (ácido lático) até níveis críticos, que resultam em ruptura da homeostase celular, precipitando a exaustão voluntária. De certa forma, os pressupostos do modelo de velocidade crítica são eminentemente energéticos/catastróficos, na medida em que explicam a exaustão em atividades severas(9) mediante aumento da taxa metabólica acima da VC de forma a provocar depleção da reserva anaeróbia. Portanto, o evento catastrófico seria a utilização total da CTA. Noakes e seus colaboradores, no entanto, apresentam fortes argumentos contra esse paradigma clássico da área. Os argumentos remontam os trabalhos seminais de A.V. Hill, e podem ser encontrados em Noakes(21), Noakes et al.(22) e principalmente em Noakes e St Clair Gibson(24).

O modelo de governador central, apresentado como alternativa aos model os catastróficos, prevê que os riscos de isquemia cardíaca e distúrbios excessivos do estado energético em nível muscular, assim como outras possíveis informações aferentes importantes, são constantemente monitorados pelo sistema nervoso central, modulando alterações na ativação neu- 
romuscular, sobretudo na iminência de qualquer dano a órgãos ou sistemas fisiológicos. Dessa forma, o exercício realizado sob hipóxia, por exemplo, induz uma menor atividade el etromiográfica no exercício máximo, bem como redução do débito cardíaco máximo(22). Essas respostas são complexas, pois envolvem tanto retroalimentação quanto pré-alimentação por parte do governador central, e a relação entre ambas determina a tolerância e a percepção ao esforço conduzido sob diversas condições.

Segundo Baldwin et al.(4), ao final de um exercício até o limite de tolerância a cerca de $70 \%$ do $\mathrm{VO}_{2} \max$, não houve redução da reserva de nucleotídeos de adenina (ATP + ADP + AMP) ou modificação na concentração dos intermediários do Ciclo de Krebs. Isso ocorreu tanto com depleção prévia ou não de glicogênio muscular. Ou seja, não houve evidência de evento "catastrófico". Em adição, segundo Noakes et al.(23), a condição com depleção prévia de glicogênio aumentou o esforço percebido a uma taxa maior que a condição sem depleção. O tempo de tolerância foi menor no primeiro. Porém, quando os dados eram plotados em relação ao percentual do tempo total de manutenção do esforço, havia sobreposição das duas funções lineares. Esse achado foi utilizado como argumento de que o esforço percebido teria capacidade de projeção, por intermédio do governador central, do tempo de tolerância até a ocorrência da exaustão. Sua principal fonte de informação, nesse caso, seriam os estoques de glicogênio remanescentes. Isso explicaria o aumento do esforço percebido nos protocolos realizados abaixo da VC que, inicialmente, não era prevista neste estudo. A maior limitação deste estudo foi o número de participantes. Portanto, ele se apresenta como estudo exploratório, já que as implicações teóricas dos resultados voltarão a ser testadas em trabal hos futuros, quiçá com atletas mais experientes e com desempenho competitivo melhor. Ainda assim, esperam-se respostas similares, pois os mecanismos de teleoantecipação em atletas com mais anos acumulados de treinamento devem ser mais consistentes. Dessa forma, os resultados do presente estudo apontam que o esforço percebido apresenta comportamento diferenciado em intensidades abaixo e acima da VC. Em treinamento intervalado com diferentes pausas ( 20 ou $40 \mathrm{~s}$ ) em intensidade inferior à VC, não há diferença na resposta perceptiva entre as repetições correspondentes. Por outro lado, em intensidade superiores à VC, interval os menores entre as repetições provocam maior percepção de esforço do que pausas mais longas, propondo como mecanismo explicativo a utilização ou não da CTA.

\section{CORRESPONDÊNCIA}

\section{Fábio Yuzo Nakamura}

Grupo de Estudo das Adaptações

Fisiológicas ao Treinamento

Centro de Educação Física e Desportos

Universidade Estadual de Londrina

Rod. Celso Garcia Cid, km 380

Campus Universitário

CEP 86051-990 - Londrina

PR - Brasil

E-mail: fabioy_nakamura@yahoo.com.br 


\section{REFERÊNCIAS}

1. Albertus $Y$, Tucker R, St Clair Gibson A, Lambert EV, Hampson, DB, Noakes T (2005). Effect of distance feedback on pacing strategy and perceived exertion during cycling. M ed Sci Sports Exerc 37:461-468.

2. Baden DA, Warwick-Evans LA, Lakomy J (2004). Am I nearly there? The effect of anticipated running distance on perceived exerinterest and attentional focus. J Sports Exerc Psychol 27: 215-31.

3. Baden DA, McLean TL, Tucker R, Noakes TD, St Clair Gibson A (2005). Effect of anticipation during unknown or unexpected exercise duration on rating of perceived exertion, affect and physiological function. Br J Sports M ed 35:742-746.

4. Baldwin J, Snow RJ, Gibala MJ, Garnham A, Howarth K, Febbraio MA (2003). Glycogen availability does not affect the TCA cycle or TAN pools during prolonged, fatigue exercise. J A ppl Physiol 94:2181-2187.

5. Borg GAV (1982). Phychophysical bases of perceived exertion. M ed Sci Sports Exerc 14:377-381.

6. Cafarelli E. Peripheral contributions to the perception of effort (1982). M ed Sci Sports Exerc 14:382-389.

7. Chatagnon M, Pouilly J-P, Thomas V, Busso T (2005) Comparasion between maximal power in the powerendurance relationship and maximal instantaneous power. Eur J A ppl Physiol 94:711-717.

8. Dekerle J, Pelayo P, Clipet B, Depretz S, Lefevre T, Sidney $M$ (2005). Critical swimming speed does not represent the speed at maximal lactate steady state. Int I Sports M ed 26:524-30.

9. Gaesser GA, Poole D (1996). The slow component of oxygen uptake kinetics in humans. Exerc Sport Sci Rev 24:3570.

10. Greco CC, Denadai BS, Pellegrinotti IL, Freitas AB, Gomide E (2003). Limiar anaeróbio e velocidade crítica determinada com diferentes distâncias em nadadores de 10 a 15 anos: relações com a performance e a resposta do lactato sanguíneo em testes de endurance. R ev Bras M ed Esporte 1:2-8.

11. Greco CC, Denadai BS (2005). Critical speed and endurance capacity in young swimmers: effects of gender and age. Pediatr Exerc Sci 17:353-63.

12. Hill DW, Smith JC (1994). A method to ensure the accuracy of estimates of anaerobic capacity derived using the critical power concept. J Sports M ed Phys Fitness 34(1):2337.

13. Hill DW, Smith JC (1999). Determination of critical power by pulmonary gas exchange. Can J A ppl Physiol 24(1): 74 86.

14. Hultman EH (1986). Carbohydrate metabolism during hard exercise and in the recovery period after exercise. Acta Physiol Scand 556:s75-82.

15. Kokubun E (1996). Velocidade crítica como estimador do limiar anaeróbio na natação. R ev Paul Ed Fis 10:5-20.

16. Martin L, Whyte GP (2000). Comparison of critical swimming velocity and velocity at lactate threshold in elite triathletes. Int J Sports Med 21:366-68.

17. Monod H, Scherrer J (1965). The work capacity of synergic muscle group. Ergonomics 8:329-338.

18. Moritani T, Nagata A, deVries HA, Muro M (1981). Critical power as a measure of physical work capacity and anaerobic threshold. Ergonomics 24:339-350.
19. Nakamura FY, Gancedo MR, Silva LA, Lima JRP, Kokubun E (2005). Utilização do esforço percebido na determinação da velocidade crítica em corrida aquática. R ev Bras M ed Esporte 11:1-5.

20. Nakamura FY, Brunetto AF, Hirai DM, Roseguini BT, Kokubun E (2005). O limiar de esforço percebido (LEP) corresponde à potência crítica e a um indicador de máximo estado estável de consumo de oxigênio. R ev Bras M ed Esporte 11:197-202.

21. Noakes TD (1997). Challenging beliefs: ex Africa semper aliquid novi. Med Sci Sports Exerc 29: 571-590.

22. Noakes TD, Peltonoen JE, Rusko HK (2001). Evidence that a central governor regulates exercise performance during acute hypoxia and hyperoxia. J Exp Biol 204: 3225-3234.

23. Noakes TD, Snow RJ, Febbraio MA (2004). Linear rela tionship between the perception of effort and the duration of constant load exercise that remains. J A ppl Physiol 96:1571-1573

24. Noakes TD, St Clair Gibson A (2004). Logical limitations to the "catastrophe" models of fatigue during exercise in humans. Br J Sports M ed 38:648-649.

25. Poole DC, Ward SA, Gardenr GW, Whipp BJ (1988). Metabolic and respiratory profile of the upper limit for prolonged exercise in man. Ergonomics. 31(9):1265-79.

26. Rauch HG, St Clair Gibson A, Lambert EV, Noakes TD (2005). A signalling role for muscle glycogen in the regulation of pace during prolonged exercise. $\mathrm{Br}$ J Sports $\mathrm{M}$ ed 39:34-38.

27. Robertson RJ, Noble BJ (1997). Perception of physical exertion: methods, mediators, and applications. Exerc Sport Sci Rev 25:407-52.

28. Seiler S, Hetleid KJ (2005). The impact of rest duration on work intensity and RPE during training. M ed Sci Sports Exerc 37:1601-1607.

29. Sjödin B, Jacobs I (1981). Onset of blood lactate accumulation and marathon running performance. Int J Sports M ed 2:23-26.

30. St Clair Gibson A, Baden BA, Lambert MI, Lambert EV, Harley YX, Hampson D, Russell VA, Noakes TD (2003). The conscious perception of sensation of fatigue. Sports M ed 33:167-176.

31. Ulmer HV (1996). Concept of anextracellular regulation of muscular metabolic rate during heavy exercise in humans by psychophysiological feedback. Experientia 52:416-420.

32. Wakayoshi K, Ikuta K, Yoshida T, Udo M, Moritani T, Mutoh Y, Miyashita M (1992). Determination and validity of critical velocity as an index of swimming performance in the competitive swimmer. Eur J A ppl Physiol 64:153-157.

33. Wakayoshi K, Yoshida T, Udo M, Kasai T, Moritani T, Mutoh Y, Miyashita M (1992). A simple method for determining critical speed as swimming fatigue threshold in competitive swimming. Int J Sports M ed 13:367-371.

34. Wakayoshi K, Yoshida T, Udo M, Harada T, Moritani T, Mutoh Y, Miyashita M (1993). Does critical swimming velocity represent exercise intensity at maximal lactate steady state? Eur J A ppl Physiol 66:90-95. 